\title{
Questes
}

\section{Les manifestations divines : éléments bibliographiques}

\section{Céline Ménager et Isabelle Coumert}

\section{(2) OpenEdition}

1 Journals

\section{Édition électronique}

URL : http://journals.openedition.org/questes/1172

DOI : 10.4000/questes. 1172

ISSN : 2109-9472

Éditeur

Les Amis de Questes

\section{Édition imprimée}

Date de publication : 15 septembre 2010

Pagination : 109-113

ISSN : 2102-7188

\section{Référence électronique}

Céline Ménager et Isabelle Coumert, «Les manifestations divines : éléments bibliographiques »,

Questes [En ligne], 19 | 2010, mis en ligne le 01 janvier 2014, consulté le 18 septembre 2020. URL

http://journals.openedition.org/questes/1172 ; DOI : https://doi.org/10.4000/questes.1172

\section{(c) Association des amis de «Questes »}




\section{Éléments bibliographiques}

\section{Piété et dévotion populaire}

Beriou, Nicole, Berlioz, Jacques, Longere, Jean (dir.), Prier au Moyen Âge. Pratiques et expériences ( $V^{e}-X V^{e}$ siècles), Turnhout, Brépols, 1991.

Cumming, Geoffrey John, BAKER, Derek (dir.), Popular Belief and Practice, Cambridge, Cambridge University Press, 1972.

Delaruelle, Étienne, La Piété populaire au Moyen Âge, Turin, Bottega d'Erasmo, 1975.

Foulon, Jean-Hervé, «L'homme face à Dieu, les temps romans, $\mathrm{XI}^{\mathrm{e}}-\mathrm{XII}^{\mathrm{e}}$ siècles, Histoire religieuse (1)», Historiens \& Géographes, $\mathrm{n}^{\circ} 341$ (numéro spécial d'Histoire religieuse) (1993), p. 187-212.

Manselli, Raoul, La Religion populaire au Moyen Âge: problèmes de méthode et d'histoire, Montréal/Paris, Institut d'histoire médiévale Albert-le-Grand/Jean Vrin, 1975.

MerdrignAC, Bertrand, La Vie religieuse en France au Moyen Âge, Gap, Ophrys, 1994.

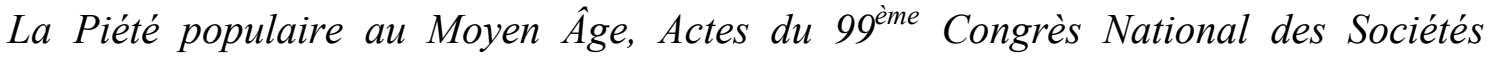
Savantes, Besançon, 1974, Paris, Bibliothèque nationale, 1977.

La Prière au Moyen Âge: littérature et civilisation, Aix-en-Provence, CUERMA Université de Provence / Paris, Honoré Champion, 1981.

TOUSSAERT Jacques, Le Sentiment religieux en Flandre à la fin du Moyen Âge, Paris, Plon, 1963.

VAuchez, André, La Spiritualité du Moyen Âge, VIII -XII siècles, Paris, Presses Universitaires de France, 1975.

VAuCHEZ, André, Les Laïcs au Moyen Âge : pratiques et expériences religieuses, Paris, Éditions du Cerf, 1987.

\section{Symbolisme et perception du merveilleux}

CHydenius, Johan, The Theory of medieval symbolism, Helsingfors, Centraltryckeriet, 1960.

Dubost, Francis, Aspects fantastiques de la littérature narrative médiévale : XII ${ }^{e}$-XIII siècles: l'autre, l'ailleurs, l'autrefois, Paris, Honoré Champion, « Nouvelle bibliothèque du Moyen Âge », 1991.

FERLAMPIN-ACHER, Christine, Merveilles et topique merveilleuse dans les romans médiévaux, Paris, Honoré Champion, 2003.

ISAMBERT, François, Rite et efficacité symbolique. Essai d'anthropologie sociologique, Paris, Éditions du Cerf, 1979.

Lecouteux, Claude, Au-delà du merveilleux : essai sur les mentalités du Moyen Âge, Paris, Presses de l'Université de Paris-Sorbonne, «Cultures et civilisations médiévales », 1998. 
LE Goff, Jacques, L’Imaginaire médiéval : essais, Paris, Gallimard, 1985.

MArtin, Hervé, Mentalités médiévales, $X I^{e}-X V^{e}$ siècles, Paris, Presses Universitaires de France, 1996.

PoIrion, Daniel, Le Merveilleux dans la littérature française du Moyen Âge, Paris, Presses Universitaires de France, « Que sais-je ? », 1982.

Rousset, Paul «Le sens du merveilleux à l'époque féodale », Moyen Âge, 62 (1956), p. 25-37.

SchmitT, Jean-Claude, La Raison des gestes dans l'Occident médiéval, Paris, Gallimard, 1990.

SCHMITT, Jean-Claude, «Rêver au XII ${ }^{\mathrm{e}}$ siècle », in Tullio GREGORY (dir.), I sogni nel Medio Evo, Rome, Edizioni dell'Ateneo, 1985, p. 291-316.

\section{Théophanie, visions et apparitions}

BARnAY, Sylvie, Le Ciel sur la terre. Les apparitions de la Vierge Marie au Moyen Âge, Paris, Éditions du Cerf, 1999.

BARNAY, Sylvie, «La mariophanie au regard de Jean de Morigny : magie ou miracle de la vision mariale? ", in Miracles, prodiges et merveilles au Moyen Âge: Actes du XXV Congrès de la Société des Historiens Médiévistes de l'Enseignement Supérieur Public d'Orléans, juin 1994, Paris, Publications de la Sorbonne, 1995.

BIELER (dir.), The Mind of Eriugena : Papers of a colloquium, Dublin, 14-18 july 1970, Dublin, Irish University Press for the Royal Irish Academy, 1973, p. 98-113.

Boespflug, François, «Un étrange spectacle: le Buisson ardent comme théophanie dans l'art occidental », Revue de l'art, 97 (1992), p. 11-31.

Christe, Yves, La Vision de Matthieu (Matth. XXIV-XXV). Origines et développement d'une image de la seconde parousie, Paris, Klincksieck, 1973.

Christe, Yves, "Quelques portails romans et l'idée de théophanie selon Jean Scot Érigène », in John O'MEARA et Ludwig BIELER (dir.), The Mind of Eriugena : Papers of a colloquium, Dublin, 14-18 july 1970, Dublin, Irish University Press for the Royal Irish Academy, 1973, p. 182-189.

CHRISTE, Yves, Les grands portails romans. Études sur l'iconologie des théophanies romanes, Genève, Droz, 1969.

Dominguez, Véronique, «Une expérience de Dieu: mystique et théâtre dans la quatrième journée du Mystère de la Passion d'Arnoul Gréban », European Medieval Drama, 5 (2001) p. 1-16.

GACHELIN, Jean-Marc, " La vision de Dieu ou la quête de l'invisible (étude de mystique médiévale) », in Danielle Buschinger et Wolfang SPIEWOK (dir.), Études de linguistique et de littérature en l'honneur d'André Crépin, Greifswalder Beiträge zum Mittelalter, 5, Wodan, 20, Greifswald, Reineke Verlag, 1993, p. 163-173.

Gros, Gérard, « L'Uis de l'arche, ou merveilleux et théophanie dans l'Estoire del Saint Graal », in Francis Gingras, Françoise LaURent, Frédérique LE NAN et Jean-René VAlette (dir.), Furent les merveilles pruvees et les aventures truvees, Paris, Honoré Champion, 2005, p. 307-320. 
HECK, Jean-Christian, « Du songe de Jacob aux visions de saints dans l'art médiéval: Théophanie et géographie sacrée. ", Micrologus : Natura, scienze e società medievali, "Vue et vision au Moyen Âge », II, 6 (1998), p. 43-57.

JEREMIAS, Jörg, Theophanie, die Geschichte einer alttestamentlichen Gattung, Neukirchener Verlag des Erziehungsvereins, Neukirchen-Vluyn, 1965.

Kessler, Herbert L., Spiritual Seeing. Picturing God's Invisibility in Medieval Art, Philadelphie, University of Pennsylvania Press, 2000.

Maillard, Pierre-Yves, La Vision de Dieu chez Thomas d'Aquin : une lecture de l'In Ioannem à la lumière de ses sources augustiniennes, Paris, Vrin, 2001.

MCGINN, Bernard, «Visio Dei : seeing God in medieval theology and mysticism », in Carolyn Muessig, Ad Putter (dir.), Envisaging Heaven in the Middle Ages, Londres, Routledge, 2007, p. 15-33.

Odonis, Gérard, Trottmann, Christian, La Vision de Dieu aux multiples formes: Quodlibet tenu à Paris en décembre 1333, Paris, Vrin, 2001.

SCRIBA, Albrecht, Die Geschichte des Motivkomplexes Theophanie: seine Elemente, Einbindung in Geschehensabläufe und Verwendungsweisen in altisraelitischer, frühjüdischer und frühchristlicher Literatur, Göttingen, Vandenhoeck \& Ruprecht, «Forschungen zur Religion und Literatur des Alten und Neuen Testaments », 167, 1995.

TORRELl, Jean-Pierre, «La vision de Dieu per essentiam selon saint Thomas d'Aquin. ", Micrologus : Natura, scienze e societa medievali, "Vue et vision au Moyen Âge », I, 5 (1997), p. 43-68.

Trouillard, Jean, « Erigène et la théophanie créatrice », in John O’MEARA et Ludwig Ludwig BIELER (dir.), The Mind of Eriugena : Papers of a colloquium, Dublin, 14-18 july 1970, Dublin, Irish University Press for the Royal Irish Academy, 1973, p. 98-113.

Trottmann, Christian, «Facies et essentia dans les conceptions médiévales de la vision de Dieu », Micrologus : Natura, scienze e societa medievali, "Vue et vision au Moyen Âge », I, 5 (1997), p. 3-18.

\section{Hagiographie et miracles}

BATAILlON, Louis-Jacques, «Les stigmates de saint François vus par Thomas d'Aquin et quelques autres prédicateurs dominicains. ", Archivum Franciscanum Historicum, 90.1-2 (1997), p. 341-347.

Boesch GAJANO, Sofia, " The use and abuse of miracles in early medieval culture », in Lester K. LitTle and Barbara H. Rosenwein (dir.), Debating the Middle Ages: Issues and Readings, Oxford, Blackwell Publishers, 1998, p. 330-339.

BRETEL, Paul, « Voies profanes et voies de Dieu : les paradigmes de la voie, de Renart aux Miracles de la Vierge », in Francis Gingras, Françoise LAURENT, Frédérique LE NAN et Jean-René VALETTE (dir.), Furent les merveilles pruvees et les aventures truvees, Paris, Honoré Champion, 2005, p. 101-122.

DiERKEns, Alain, "Réflexions sur le miracle au haut Moyen Âge », in Miracles, prodiges et merveilles au Moyen Âge: Actes du XXV Congrès de la Société des Historiens Médiévistes de l'Enseignement Supérieur Public d'Orléans, juin 1994, Paris, Publications de la Sorbonne, 1995, p. 9-30. 
Duchesne, Annie, "Miracles et merveilles chez Gervais de Tilbury », in Miracles, prodiges et merveilles au Moyen Âge: Actes du XXV Congrès de la Société des Historiens Médiévistes de l'Enseignement Supérieur Public d'Orléans, juin 1994, Paris, Publications de la Sorbonne, 1995, p. 151-158.

Les Fonctions des saints dans le monde occidental (III $-X I I I^{e}$ siècles), Actes $d u$ colloque, Rome, 27-29 octobre 1987, Rome, EFR, 1991.

ForThomme, Bernard, «Le signe jaloux des stigmates et ses contestations », Antonianum Periodicum philosophico-theologicum trimestre, 78.2 (2003), p. 249-290.

Goodich, Michael, Miracles and Wonders. The Development of the Concept of Miracle, 1150-1350, Aldershot, Ashgate, 2007.

Holdsworth, Christopher, "Reading the signs: Bernard of Clairvaux and his miracles ", in David BATES, Julia CRICK et Sarah HAMILTON (dir.), Writing Medieval Biography, 750-1250, Woodbridge, Suffolk, The Boydell Press, 2006, p. 161-172.

Justice, Steven, « Did the Middle Ages believe in their miracles? », Representations, 103 (2008), p. 1-29.

Miracles, prodiges et merveilles au Moyen Âge, XXV congrès SHMESP, Orléans, 1994, Paris, Publication de la Sorbonne, 1995.

Parish, Helen I., Monks, Miracles and Magic. Reformation Representations of the Medieval Church, Londres, Routledge, 2005.

RASSINIER, Jean-Paul, "Miracles et pathologie dans l'œuvre de saint Augustin », in Bernard Ribemont (dir.), Le Corps et ses énigmes au Moyen Âge. Actes du Colloque, Orléans, 15-16 mai 1992, Caen, Paradigme, 1993, p. 133-156.

Riche, Pierre puis VAuchez, André (dir.), Histoire des saints et de la sainteté chrétienne, Paris, Hachette, 1986.

SCHMITT, Jean-Claude, «Religion et guérison dans l'Occident médiéval », in Historiens et sociologues aujourd'hui, Journées d'Études annuelles de la Société Française de Sociologie, Université de Lille I (14-15 juin 1984), Paris, CNRS, 1986, p. 135-150.

Sigal, Pierre-André, L'Homme et le miracle en France aux XI et XII siècles, Paris, Éditions du Cerf, 1985.

SigNORET, Jean-Olivier, Deo gubernante : navigations miraculeuses et miracles marins au Moyen Âge, l'union des cultures païennes et chrétiennes, Villeneuve d'Ascq, Presses Universitaires du Septentrion, 2003.

VAlette, Jean-René, «Miracle et merveille dans les proses du Graal », in Francis Gingras, Françoise Laurent, Frédérique Le NAN et Jean-René VALETTE (dir.), Furent les merveilles pruvees et les aventures truvees, Paris, Honoré Champion, 2005, p. 673696.

VAUCHEZ, André, «Les stigmates de saint François et leurs détracteurs dans les derniers siècles du Moyen Âge ", in André VAucheZ, Religion et société dans l'Occident médiéval, Turin, Bottega d'Erasmo, 1980, p. 139-169.

VAuchez, André, La Sainteté en Occident aux derniers siècles du Moyen Âge, d'après les procès de canonisation et les documents hagiographiques, Rome, EFR, 1981.

VAUCHEZ, André, Saints, prophètes et visionnaires. Le pouvoir surnaturel au Moyen Âge, Paris, Albin Michel, 1999. 
WARD, Benedicta, Miracles and the Medieval Mind: Theory, Record and Event (10001215), Philadelphia, University of Pennsylvania Press / London, Scolar press, 1982.

\section{Autres manifestations}

BARTLETt, Robert, Trial by Fire and Water. The Medieval Judicial Ordeal, Oxford, Clarendon, 1986.

BERLIOZ, Jacques, « Les récits exemplaires et l'histoire des catastrophes naturelles », in Jacques Berlioz, Catastrophes naturelles et calamités au Moyen Âge, Florence, SISMEL, 1998, p. 33-55.

COONEY, Helen, « Wonder and immanent justice in the Man of Law's Tale », Chaucer Review, A Journal of Medieval Studies and Literary Criticism, 33 (1999), p. 264-287.

GAudemet, Jean, «Les ordalies au Moyen Âge », Recueils de la société Jean Bodin, XVII, 2 (1965), p. 99-135.

KAMMERER, Odile, «Un prodige en Alsace à la fin du $\mathrm{XV}^{\mathrm{e}}$ siècle : la météorite d'Ensisheim ", in Miracles, prodiges et merveilles au Moyen Âge: Actes du XXV Congrès de la Société des Historiens Médiévistes de l'Enseignement Supérieur Public d'Orléans, juin 1994, Paris, Publications de la Sorbonne, 1995, p. 294-315.

NABERT, Nathalie, "Climat, saisons, phénomènes atmosphériques dans les psaumes », in Joëlle Ducos et Claude Thomasset (dir.), Le Temps qu'il fait au Moyen-Âge: phénomènes atmosphériques dans la littérature, la pensée scientifique et religieuse, Paris, Presses de l'Université de Paris-Sorbonne, «Cultures et Civilisations Médiévales », 1998, p. 171-190. 